\title{
Effects of a dietary intervention promoting the adoption of a Mediterranean food pattern on fast-food consumption among healthy French-Canadian women
}

\author{
Alexandra Bédard ${ }^{1}$, Julie Goulet ${ }^{2}$, Mélissa Riverin ${ }^{1}$, Benoît Lamarche ${ }^{1}$ and Simone Lemieux ${ }^{1 *}$ \\ ${ }^{1}$ Institute of Nutraceuticals and Functional Foods, Laval University, 2440 Hochelaga Blvd, QC, Canada G1V 0A6 \\ ${ }^{2}$ Canadian Population Health Initiative/Canadian Institute for Health Information, 495 Richmond Road, Suite 600, Ottawa ON \\ Canada K2A $4 H 6$
}

(Received 30 November 2009 - Revised 23 April 2010 - Accepted 1 June 2010 - First published online 9 August 2010)

It is expected that a dietary intervention based on the traditional Mediterranean food pattern should be associated with a reduction in fast-food consumption but this has never been tested before. We assessed the impact of a 12-week dietary intervention, promoting the adoption of a Mediterranean food pattern, on fast-food consumption among seventy-one healthy women aged between 30 and 65 years. The dietary intervention consisted of two group sessions and seven individual sessions with a dietitian. To determine the Mediterranean dietary score (MedScore) and fast-food consumption, an FFQ was administered. During the 12-week intervention, the MedScore significantly increased (from 21.1 (SD 3.6) units at baseline to 28.6 (SD 4.4) units at week 12, $P<0.0001$ ), while the fast-food consumption significantly decreased (from 51.7 (SD 46.4 ) g/d at baseline to 20.5 (SD 18.2) g/d at week $12, P<0.0001)$. Moreover, women who had a higher consumption of fast food at baseline decreased their fast-food consumption to the most $(r-0.50, P<0.0001)$. When four subgroups were formed on the basis of median values of Medscore and fast-food consumption changes, it was found that only the subgroup of women which increased the most their MedScore and decreased the most their fast-food consumption experienced a significant decrease in BMI $(P<0 \cdot 01)$. In conclusion, a dietary intervention promoting the Mediterranean food pattern led to a decrease in fast-food consumption among healthy women even if it was not a specific target of the intervention. Dietary strategies for increasing intake of healthy foods may be a useful approach for decreasing intake of less healthy foods.

Mediterranean food pattern: Fast-food consumption: BMI: Lipid profile

In the past 30 years, a dramatic increase in fast-food consumption has occurred. Several studies, supported by a recent review of the literature, suggest that the increase in fast-food consumption may be one of the important contributors of remarkable increases in overweight and obesity rates in the past decades ${ }^{(1,2)}$. In fact, fast food is characterised by a high energy density ${ }^{(3)}$, a high palatability ${ }^{(4)}$ and a poor content in dietary fibres ${ }^{(3)}$, and all these factors have been associated to promote an increase in energy intake ${ }^{(4-6)}$. Moreover, some evidence showed that fast-food consumption is also related to some cardiovascular risk factors ${ }^{(7,8)}$.

To counter the increased rates of obesity and related diseases, many organisations recently recommended low energy density food patterns such as the Mediterranean diet ${ }^{(9)}$. In contrast to fast food, the Mediterranean food pattern that contains high amounts of antioxidants, dietary fibres and unsaturated fats may have protective effects on CVD and may prevent weight gain $^{(10,11)}$.

On the other hand, some studies have shown that intervening on specific diet components can bring dietary benefits other than those associated with the primary intervention target. As an example, it has been shown that an intervention promoting a high consumption of fruits and vegetables may lead to a decrease in the intake of foods rich in fats and sugars ${ }^{(12)}$. Thus, it is expected that a dietary intervention promoting the adoption of a Mediterranean food pattern should be associated with a reduction in fast-food consumption but this has never been tested before. Therefore, the main purpose of the present study was to determine the effect of a dietary intervention promoting the Mediterranean food pattern on fast-food consumption among healthy French-Canadian women under free-living conditions.

\section{Subjects and methods}

\section{Subjects}

The study sample included healthy women, 30-65 years of age, living in Québec city metropolitan area. Potential candidates were selected on the basis of the following eligibility criteria: absence of metabolic disorders requiring treatment; stable body weight for at least 3 months before the start of the study; being in charge of food purchases and meal preparation most of the time and not consuming a diet concordant with Mediterranean food pattern at the beginning of the study. Of the 126 women examined at the 
screening, ninety-four met the above-mentioned criteria and seventy-seven took part in the study. During the intervention, three women left the study for personal reasons and two women had incomplete data. Therefore, seventy-two women were included in these analyses. The present study was conducted according to the guidelines laid down in the Declaration of Helsinki, and all the procedures involving human subjects were approved by the Ethics Committees of Laval University. Written informed consent was obtained from all the subjects.

\section{Intervention}

A detailed description of the intervention has been published elsewhere ${ }^{(13)}$. Briefly, the 12-week intervention included two group and seven individual sessions with a dietitian. The objective of the first group session was to explain the principles of the Mediterranean food pattern and its health benefits. During the second group session, women had a cooking lesson in which they had to produce a complete meal based on the Mediterranean food pattern. The goal of individual sessions was to evaluate the dietary changes and to select further personalised objectives to increase their adherence to the Mediterranean food pattern. Decreasing fast-food consumption was not a specific target of the intervention.

\section{FFQ, Mediterranean dietary score (MedScore) and fast-food consumption}

A validated quantitative FFQ was administered by a registered dietitian at baseline and then at weeks 6 and 12. Details about validity and reproducibility of this FFQ have been previously reported $^{(14)}$. The MedScore, created on the basis of the Mediterranean pyramid, varied between 0 and 44 points. A MedScore of 44 would imply that food pattern is perfectly concordant with the traditional Mediterranean food pattern ${ }^{(13)}$.

The dietary-group labelled 'fast food' included hamburgers, nuggets, hot-dogs. French fries, 'poutine' (a typical FrenchCanadian dish made of French fries, cheese and gravy), fried chicken, club sandwich, hot chicken, pizza and potato chips. The amount of fast food consumed by each participant was estimated in grams by adding the usual portion size of every item included in this food group reported in the FFQ at weeks 0,6 and 12 . We chose to calculate fast-food consumption in grams to determine more precisely the associations between fast-food consumption and other quantitative variables, as performed in a previous study ${ }^{(1)}$.

\section{Clinical and laboratory measurements}

Anthropometric measurements were performed (BMI and waist circumference), and blood samples were collected at weeks 0,6 and 12 to measure TAG, total cholesterol, HDL-cholesterol, LDL-cholesterol (LDL-C), apo B, oxidised LDL-C and integrated LDL-C particle size, which corresponds to the weighted mean size of all LDL-C subclasses in an individual $^{(13,15,16)}$.

\section{Statistical analyses}

Data are presented as means and standard deviations. Pearson correlations were used to evaluate the association between changes in different variables during the 12-week intervention. For variables not normally distributed, a log-transformation was performed. Mixed procedures for repeated measurements were performed to assess the effects of time on MedScore and fast-food consumption during the intervention.

To evaluate the relative contribution of MedScore and fastfood consumption changes during the intervention on changes in anthropometric variables, participants were separated into four subgroups according to the medians of changes for fast-food consumption $(-19.3 \mathrm{~g} / \mathrm{d})$ and for MedScore $(+7$ arbitrary units). Mixed procedures for repeated measurements were used to evaluate group, time and group-by-time interaction effects. Tukey-Kramer adjusted $P$ values were used to determine statistical significant differences between and among subgroups when a significant effect was detected by the Mixed analyses. All the analyses were performed with the SAS statistical package version 9.1 (SAS Institute, Inc., Cary, NC, USA) and a $P \leq 0.05$ was considered as significant. One woman displayed extreme data at baseline in total energy intake (18 $543 \mathrm{~kJ}$ (4432 kcal)). This woman was clearly an outlier for many dietary variables and was therefore excluded from these analyses.

\section{Results}

As shown in Fig. 1, the dietary intervention promoting the Mediterranean food pattern resulted in a significant increase in MedScore after 6 weeks $(P<0 \cdot 0001)$ with no further significant change at week $12(P=0.63)$. Moreover, fast-food consumption was significantly lower after $6(P<0.0001)$ and $12(P<0.0001)$ weeks of intervention than at baseline. No significant change in fast-food intake occurred between weeks 6 and $12 \quad(P=0 \cdot 78)$. The change in fast-food consumption was correlated neither to the change in MedScore $(r 0.05, P=0.69)$ nor to any of its individual components (results not shown).

A significant negative association was observed between fast-food consumption at baseline and change in fast-food consumption between week 0 and week 12 ( $r-0 \cdot 50$, $P<0.0001)$. Fast-food consumption change was not correlated

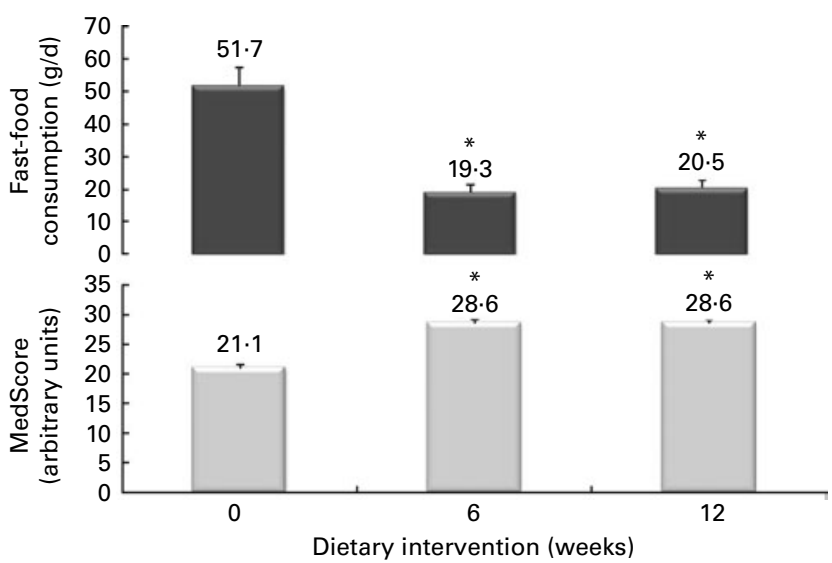

Fig. 1. Fast-food consumption and MedScore at different time points during the 12-week dietary intervention. Data are presented as mean values with their standard errors ( $n$ 71). Data were analysed using Mixed procedures followed by the Tukey-Kramer test. ${ }^{*}$ Mean value was significantly different from that at week $0(P<0.05)$. 
with changes in anthropometric variables. However, after the formation of four subgroups based on the medians of change in MedScore and change in fast-food consumption during the intervention, a significant decrease in BMI was observed only in the subgroup of women with a larger increase in MedScore and a larger decrease in fast-food consumption in response to the intervention $(P=0 \cdot 001)$ (Table 1$)$.

With regard to metabolic variables, change in fast-food consumption was associated with changes in total cholesterol concentration, LDL-C concentration and integrated LDL-C particle size $(r 0.29, P=0.01 ; r 0.25, P=0.04$ and $r 0.35$, $P=0.002$, respectively). However, it was not significantly associated with changes in concentrations of TAG, HDL-cholesterol, apo B and oxidised LDL-C.

\section{Discussion}

To the best of our knowledge, this is the first interventional study showing that a dietary intervention promoting the Mediterranean food pattern results, in addition to improved adherence to this food pattern, in a significant decrease in fast-food consumption among healthy women in free-living conditions.

In concordance with our findings, results from a crosssectional study showed that subjects who consumed meals at fast-food restaurants more than once per week had a lower adherence to the traditional Mediterranean food pattern than subjects who consumed meals at fast-food restaurants less frequently ${ }^{(1)}$. However, in our sample, the reduction in fast-food consumption was directly correlated neither with the increase in the adherence to the Mediterranean food pattern nor with any of the MedScore components. In fact, results showed that change in fast-food consumption was mainly influenced by fast-food consumption at baseline. Thus, these results suggest that regardless of the change in the adherence to the Mediterranean food pattern, women characterised by a higher fast-food consumption at baseline had more room for improvement with regard to this food habit than women who consumed a smaller amount of fast food.

Further analyses also revealed that a significant decrease in BMI was observed only in the subgroup of women who had both a larger increase in MedScore and a larger decrease in fast-food consumption. These results are in line with other studies which showed that an increase in the adherence to the Mediterranean food pattern and a decrease in fast-food consumption are both associated with a decrease in energy density $^{(3,17)}$. A decrease in energy density has been shown to be associated with a reduction in energy intake ${ }^{(18)}$. Thus, the present study results suggest that an increase in MedScore along with a decrease in fast-food consumption was required to decrease energy density sufficiently for inducing body weight loss during the intervention.

Associations between change in fast-food consumption and changes in total cholesterol concentration, more precisely LDL-C concentration, are concordant with other studies ${ }^{(19)}$. Moreover, in the present study, a decrease in integrated LDL-C particle size was associated with a decrease in fast-food consumption. This deleterious effect in women who decreased fast-food consumption is in line with the results of Kratz et al. ${ }^{(20)}$ who observed that, compared with a diet rich in SFA, such as fast food, a diet rich in unsaturated

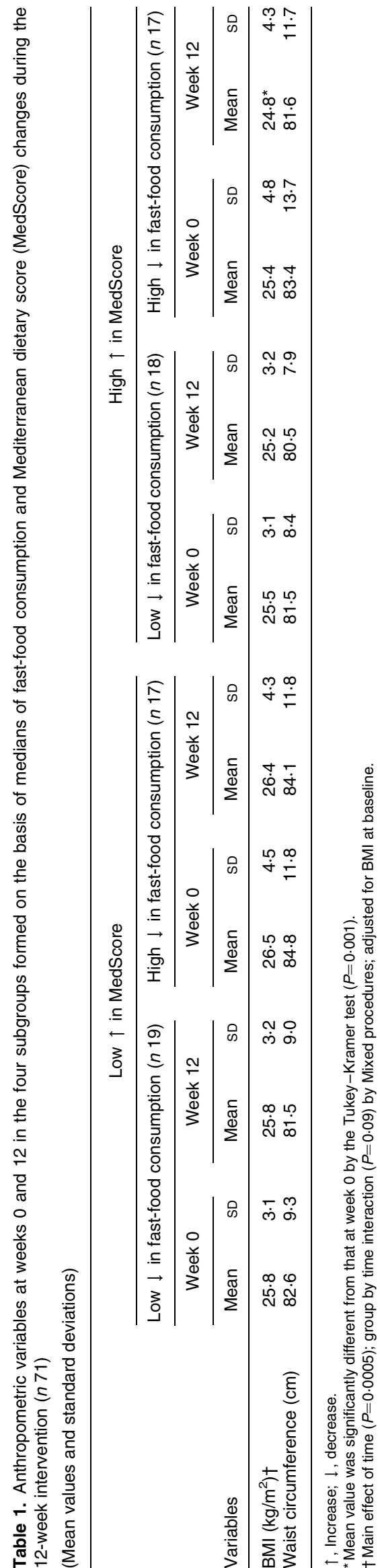


fatty acids led to a reduction in LDL-peak particle diameter (i.e. diameter of the most predominant LDL subclass). It remains to be established if this small decrease in integrated LDL-C particle size may cause deleterious effects on cardiovascular health in the long term.

We acknowledge that the present study has some limitations such as the lack of a control group. The inclusion of a control group would have helped us to determine whether the decrease in fast-food consumption was related to the intervention or was explained by a study effect. On the other hand, strengths of the study include the intense nature of the follow-up and the efforts to ensure standardisation as well as the inclusion of biochemical measures.

In summary, a dietary intervention promoting the Mediterranean food pattern under free-living conditions resulted in a significant decrease in fast-food consumption among healthy women, though it was not a specific target of the dietary intervention. These results suggest that focusing on the increasing intake of healthy foods may be a useful approach for decreasing intakes of less healthy foods.

\section{Acknowledgements}

A. B. and M. R. analysed the data and A. B. wrote the manuscript. J. G. supervised the study and reviewed the manuscript. B. L. and S. L. designed the study, supervised the study and reviewed the manuscript. This research received no specific grant from any funding agency in the public, commercial or not-for-profit sectors. Authors report no conflict of interest.

\section{References}

1. Schroder H, Fito M \& Covas MI (2007) Association of fast food consumption with energy intake, diet quality, body mass index and the risk of obesity in a representative Mediterranean population. Br J Nutr 98, 1274-1280.

2. Rosenheck R (2008) Fast food consumption and increased caloric intake: a systematic review of a trajectory towards weight gain and obesity risk. Obes Rev 9, 535-547.

3. Bowman SA, Gortmaker SL, Ebbeling CB, et al. (2004) Effects of fast-food consumption on energy intake and diet quality among children in a national household survey. Pediatrics 113, 112-118.

4. Blundell JE \& MacDiarmid JI (1997) Fat as a risk factor for overconsumption: satiation, satiety, and patterns of eating. J Am Diet Assoc 97, S63-S69.

5. Bell EA, Castellanos VH, Pelkman CL, et al. (1998) Energy density of foods affects energy intake in normal-weight women. Am J Clin Nutr 67, 412-420.

6. Linde JA, Utter J, Jeffery RW, et al. (2006) Specific food intake, fat and fiber intake, and behavioral correlates of BMI among overweight and obese members of a managed care organization. Int J Behav Nutr Phys Act 3, 42.
7. Brunner H, Cockcroft JR, Deanfield J, et al. (2005) Endothelial function and dysfunction. Part II: Association with cardiovascular risk factors and diseases. A statement by the Working Group on Endothelins and Endothelial Factors of the European Society of Hypertension. J Hypertens 23, 233-246.

8. Burdge GC \& Calder PC (2005) Plasma cytokine response during the postprandial period: a potential causal process in vascular disease? Br J Nutr 93, 3-9.

9. Klein S, Sheard NF, Pi-Sunyer X, et al. (2004) Weight management through lifestyle modification for the prevention and management of type 2 diabetes: rationale and strategies. A statement of the American Diabetes Association, the North American Association for the Study of Obesity, and the American Society for Clinical Nutrition. Am J Clin Nutr 80, 257-263.

10. Sanchez-Tainta A, Estruch R, Bullo M, et al. (2008) Adherence to a Mediterranean-type diet and reduced prevalence of clustered cardiovascular risk factors in a cohort of 3,204 high-risk patients. Eur J Cardiovasc Prev Rehabil 15, 589-593.

11. Panagiotakos DB, Chrysohoou C, Pitsavos C, et al. (2006) Association between the prevalence of obesity and adherence to the Mediterranean diet: the ATTICA study. Nutrition 22, 449-456.

12. Epstein LH, Gordy CC, Raynor HA, et al. (2001) Increasing fruit and vegetable intake and decreasing fat and sugar intake in families at risk for childhood obesity. Obes Res 9, 171-178.

13. Goulet J, Lamarche B, Nadeau G, et al. (2003) Effect of a nutritional intervention promoting the Mediterranean food pattern on plasma lipids, lipoproteins and body weight in healthy French-Canadian women. Atherosclerosis 170, 115-124.

14. Goulet J, Nadeau G, Lapointe A, et al. (2004) Validity and reproducibility of an interviewer-administered food frequency questionnaire for healthy French-Canadian men and women. Nutr J 3, 13.

15. Lapointe A, Goulet J, Couillard C, et al. (2005) A nutritional intervention promoting the Mediterranean food pattern is associated with a decrease in circulating oxidized LDL particles in healthy women from the Quebec City metropolitan area. J Nutr 135, 410-415.

16. Goulet J, Lamarche B, Charest A, et al. (2004) Effect of a nutritional intervention promoting the Mediterranean food pattern on electrophoretic characteristics of low-density lipoprotein particles in healthy women from the Quebec City metropolitan area. Br J Nutr 92, 285-293.

17. Schroder H, Vila J, Marrugat J, et al. (2008) Low energy density diets are associated with favorable nutrient intake profile and adequacy in free-living elderly men and women. $J$ Nutr 138, $1476-1481$.

18. Ello-Martin JA, Ledikwe JH \& Rolls BJ (2005) The influence of food portion size and energy density on energy intake: implications for weight management. Am J Clin Nutr 82, 236S-241S.

19. Hegsted DM, Ausman LM, Johnson JA, et al. (1993) DietaryFat and Serum-Lipids - an evaluation of the experimentaldata. Am J Clin Nutr 57, 875-883.

20. Kratz M, Gulbahce E, von Eckardstein A, et al. (2002) Dietary mono- and polyunsaturated fatty acids similarly affect LDL size in healthy men and women. $J$ Nutr 132, 715-718. 